\title{
Sub-scale models and infrastructure for accelerator magnet development
}

\author{
Snowmass 2021 : Contribution August 2020
}

\author{
Stoyan Stoynev \\ APS-TD, Fermi National Accelerator Laboratory, Batavia, Illinois 60510, USA \\ e-mail: stoyan@fnal.gov
}

Date: 25 August 2020

The LHC is undoubtfully the most important instrument the HEP community has on its disposal and is widely considered an amazing technological achievement. At the core of this instrument lay accelerator magnets based on NbTi technology - a technology well developed and widely used in the commercial sector too. Yet, a decade after the accelerator started it still did not reach its design collision energy of $14 \mathrm{TeV}$ [1] and it operated for years well below it. The reason for this is simple - magnet training [2], [3] (even though only a subset of the magnets is mostly affected).

LHC upgrades and future colliders rely on new technologies allowing to reach higher operational fields and the most practical one now is based on $\mathrm{Nb}_{3} \mathrm{Sn}$ conductor. $\mathrm{Nb}_{3} \mathrm{Sn}$ superconducting properties were in fact discovered 7 years before $\mathrm{NbTi}$. There are good reasons its development got delayed, in particular its mechanical properties are much worse than the very strong $\mathrm{NbTi}$. As a result, $\mathrm{Nb}_{3} \mathrm{Sn}$ accelerator magnets of today, as a rule, show an unpleasant feature - much longer training.

How come after decades of magnet development we are still unable to successfully deal with the problem of magnet training? The problem was known and acknowledged many decades ago [4], there were and are continuous attempts to solve it [5], [6], [7], [8], it is a top priority goal of the MDP [9]. Based on the many years of experience do we have the confidence it will be resolved, how?

Magnet training is a complex phenomenon of a complex and expensive object. What we do so far is fabricating sub-scale magnets/prototypes of the order of onetwo meters long, with otherwise the same structure of a long magnet. Sometimes a coil(s) is(are) replaced by iron block(s). All this works well for development but those are still expensive objects, we can build limited number of them, fabricating and testing them takes years and, yes, we still have problems figuring out the training. In fact, there is no firm understanding of what the exact causes are and how to mitigate them although there are some concrete yet partial successes [10].

It is arguably the case that throughout the human history people tried to resolve complex problems by first simplifying them, building "toy experiments", understanding the basics from something that is comprehensible before elaborating to structures of great complexity. It is arguably the case we should also consider all our development options, while resolving other technological difficulties.

Magnet training is the continuous increase of maximum current the magnet reaches during ramps before a natural quench occurs. It eventually reaches a plateau, 
eventually at its design level. The training is associated to the magnet coils which are seemingly trained independently in magnets [11]. Sub-scale coils are considered the smallest models for magnet development. However, multiple consecutive training quenches do happen in the same coil locations and are even responsible for long stretches of the training curve if not defining it [12]. It is plausible and even logical to consider that the phenomenon could be driven by changes in local conditions alone. In that case a "sub-scale" model of those local conditions may be well suited to explore the phenomenon and pinpoint the underlying reasons for it, possibly opening the way to mitigate their effects.

The local conditions of interest involve interface boundaries, force and magnetic fields, temperature and current through the conductor. One can imagine "cutting" piece of the magnet, supplying current through a single cable "turn" and immersing it in cooling bath under controlled force and magnetic fields to reconstruct the original conditions. While this is not an easy task it is not outright impossible to realize. There are devices for cable testing in external magnetic field under external pressure (like FRESCA2 [13]). What is needed is a more elaborate pressure device where we can test cables with interfaces, a.k.a. "cable stacks", ideally under variable force vector that can be controlled in concert with an external magnetic field vector. The initial goal is not to create precise magnet conditions, rather it is to achieve control over "training" in those "cable stacks", understanding fully the underlying reasons there. It is assumed, though complete itemization requires concrete design, that a "cable stack" fabrication is in average much cheaper than magnet fabrication, we can achieve good statistics with reproducible samples (both of which are of extreme importance) and the testing cycles are much shorter. Those are the characteristics we can not reasonably achieve in magnets and thus our studies consistently suffer. Over time we do want to extend the studies to reproducing magnet conditions in "cable stacks" precisely and it will be an effort to prove "training in magnet stacks" can be representative of "training in magnet coils" even if in limited cases. The leading principle to prove and base consequent studies on is that the phenomenon of training is basically a local phenomenon though the locality could shift in more complex objects. It is clear that if "cable stacks" are representative models, at least for training, they can be used for magnet technology development (for instance but not limited to epoxy studies) serving as simple test vehicles before building a real coil.

To be fair, there were training tests on cables [14], there were tests conducted even on wires (for a subject review and references see [15]). No dedicated devices were ever built. Pressure devices for wires (that is, a single strand is typically powered) inside external magnetic fields can serve as models or may be prototypes for "cable stack device" fabrication [16], or FRESCA2 [13] type devices can be modified. We need to put some thinking on devising a dedicated device that can serve the purpose - synchronous control of force and magnetic vector-fields on a modifiable test sample immersed in cooling bath. Then we have a chance to avoid stumbling on the same problem again-and-again by systematically studying it at our terms. 


\section{References}

[1] "LHC prepares for new achievements", https://home.cern/news/pressrelease/accelerators/Ihc-prepares-new-achievements, 3 December 2018. Retrieved 24 July 2020.

[2] E. Todesco et al., "Training Behavior of the Main Dipoles in the Large Hadron Collider", IEEE Trans. Appl. Supercond. , vol. 27, no. 4, 4702807, June 2017.

[3] "Restarting the LHC: Why 13 Tev?", https://home.cern/science/engineering/restarting-lhc-why-13-tev, Retrieved 24 July 2020.

[4] P.F. Smith and B. Colyer, "A Solution to the Training Problem in Superconducting Magnets", Cryogenics 15 (1975) 201-207.

[5] Charlie Sanabria, https://conferences.lbl.gov/event/204/

"An experiment to explore training behavior in superconducting magnets - Part 1: solenoid fabrication", https://conferences.lbl.gov/event/204/ (2018), presented.

[6] Helene Felice et al., "Design and Test of a Nb3Sn Subscale Dipole Magnet for Training Studies", IEEE Trans.Appl.Supercond. 17 (2007) no.2, 1144-1148.

[7] C. Kovacs et al., "A Cable-Scale Experiment to Explore New Materials for Optimizing Superconductor Accelerator Magnets", Cryogenics, 106:103025, 2019.

[8] Charlie Sanabria, https://conferences.lbl.gov/event/204/

"An experiment to explore training behavior in superconducting magnets - Part 1: solenoid fabrication", https://conferences.lbl.gov/event/204/ (2018), presented.

[9] "The U.S. Magnet Development Program Plan", https://atap.lbl.gov/wpcontent/uploads/sites/22/2016/01/MagnetDevelopmentProgramPlan.pdf, Retrieved 24 July 2020.

[10] D. Arbelaez et al., "Design and test results of the Nb3Sn canted-cosine-Theta dipole magnet CCT5," presented at the Appl. Supercond. Conf., Seattle, WA, USA, Oct. 28-Nov. 2, 2018, Paper 2LOr1B-04.

[11] S. Stoynev et al., "Analysis of Nb3Sn Accelerator Magnet Training" ", IEEE Trans. Appl. Supercond., , vol. 29, no. 5, 4001206, 2019.

[12] T. Strauss et al., "Quench Location in the LARP MQXFS1 Prototype", IEEE Trans. Appl. Supercond., , vol. 28, no. 3, 4001604, 2018.

[13] Willering, G.; Arnestad, H.; Bajko, M.; Bajas, H.; Bortot, L.; Bottura, L.; Bourcey, N.; Duda, M.; Ferracin, P.;Feuvrier, J.; et al., "Tests of the FRESCA2 $100 \mathrm{~mm}$ bore Nb3Sn blockcoil magnet to a record field of 14.6 T.", IEEE Trans. Appl. Supercond. 2019, 29, 4004906. [14] Ghosh, A.K., Garber, M., Robins, K.E., and Sampson, W.B. Mon . "Training in test samples of superconducting cables for accelerator magnets". United States. https://www.osti.gov/servlets/purl/6356510.

[15] Amaud DEVRED, "QUENCH ORIGINS", AIP Conference Proceedings 249, 1262 (1992); https://doi.org/10.1063/1.41993

[16] E. Barzi, M. Fratini, A. V. Zlobin, "A Device to Test Critical Current Sensitivity of Nb3Sn Cables to Pressure", Advances in Cryogenic Engineering, V. 48, AIP, V. 614, pp. 45-52 (2002). 